\title{
Improving allergen concentration homogeneity for investigating the effect of corona discharge on Der p1 allergen
}

\author{
Neil Goodman*, John F. Hughes \\ Bioelectrostatics Research Centre, Department of Electronics and Computer Science, Faraday Building \\ (Building 21), University of Southampton, Highfield, Southampton, UK SO 171 BJ
}

Received 8 June 2001; received in revised form 30 October 2001; accepted 1 November 2001

\begin{abstract}
Air ionisers utilising corona discharge have been commercially available for some time, although clinical trials have shown that these do not improve the health of asthma sufferers. New research has shown that the corona products can actually destroy allergens including the main allergen of the house dust mite Dermatophagoides pteronyssinus; Der p1. Concentration heterogeneity of commonly used sources of allergen and the additional problems that occur with electrostatic studies mean that present methods of allergen preparation are unsatisfactory. This paper describes work that improves the repeatability of tests investigating the effects of ionic bombardment on Der p1 allergen concentration. Initial experimental methods involved the distribution of sieved culture medium over a surface and divided into sectors for concentration analysis but this gave extremely variable data and so any effect of an allergen reduction method could not reliably be assessed. Different methods of distributing the sample are described in this paper. The results show that by dissolving Der p1 samples and using small evaporated aliquots, samples of homogenous concentration can be reliably used in electrostatic investigations. The benefits of using the new evaporated solution method is described with a comparison made between the effect of negative corona discharge on Der p1 using heterogeneous Der p1-containing culture particles and the new technique described in the present paper. Using the new technique of sample preparation, reductions in the Der $\mathrm{p} 1$ concentration increased from a significant $(P<0.01) 37.26 \%$ after $60 \mathrm{~min}$ to $67.37 \%$ after $300 \mathrm{~min}$. Using faecal particles, however, led to insignificant $(P>0.05)$ reductions that ranged from $4.11 \%$ to $26.86 \%$ after 60 and $300 \mathrm{~min}$, respectively. (C) 2002 Elsevier Science B.V. All rights reserved.
\end{abstract}

Keywords: Dust mites; Asthma; Air ionizer; Corona; Denaturing; Allergen

*Corresponding author. Tel.: +44-2380593703; fax: +44-2380593709.

E-mail address: goodman_neil@hotmail.com (N. Goodman). 


\section{Introduction}

Over the past 20-30 years the incidence of bronchial asthma has steadily increased in almost every country around the globe [1]. In parallel to this there have been reports of up to 5-fold increases in the prevalence of house dust mites (HDMs) in random samples collected over a 10-year period from homes in both temperate [2] and warmer [3] climates. As the link between HDMs and the occurrence of asthma and other atopic diseases is now well established [4], methods of reducing exposure to HDM allergens is of the utmost importance in the avoidance and control of these diseases.

Der $\mathrm{p} 1$ is the main allergen of the HDM Dermatophagoides pterynissinus. It is a digestive enzyme that is secreted into the mite's alimentary canal [5], which is the reason why $95 \%$ of all dust mite allergen is associated with the faecal particles [6]. Der $\mathrm{p} 1$ is a protein that causes the cleavage of other proteins, the activity of which is thought to be related to its immunogenicity. In other words, Der p1 is an allergen partly because of its functionality. As the protein is functional due to its tertiary structure (which is due in part to electrostatic forces) the breakdown of this structure by altering, or interfering with its charges may modify its functionality. Allergencarrying faecal particles have been found to tribo-charge predominantly to a positive polarity [7]. This positive charge was retained even after prolonged contact with a grounded surface, which suggested that the charge was inherent to the protein carried on the faeces. Analysis of the cDNA sequence has produced the sequence of amino acids that make up the Der p1 protein [8]. If the number of positive and negative charges is counted, it can be seen that the protein has a net +3 charge.

Previous research into the use of air ionisers for asthma sufferers has shown little to no evidence for any clinical benefit [9]. In some cases the condition of the patient was even worsened [10]. It had been thought that the electrostatic precipitation of airborne particulates by ionisers would benefit sufferers by reducing the level of allergen and dust in the air leading to less being inhaled. However, the corona products are harmful to airways and can lead to inflammation, worsening of symptoms and even prime the allergen-induced responses in the airways [11]. New research has shown that corona products can be used to destroy allergens [12]. With a revised protocol for use, air ionisers may have an application in the domestic environment to clean the house of allergy-causing proteins in situ before they can harm the body.

To investigate the effects of corona discharge, a novel and improved method of allergen sample preparation was required. The standard method of investigating the effects of chemical or physical treatments on domestic allergens is to use house dust, HDM culture, or a mixture of both. This method has the advantage of mimicking in situ characteristics such as interactions with other dust components, and retention in carpeting and soft furnishings, etc. However, when investigating the effects of either a chemical or physical treatment on allergens, the use of heterogeneous powder mixtures inevitably leads to concentration variability between samples. Such variability means that the exact concentration of the samples tested cannot be known precisely. This could lead to difficulties in distinguishing the effects of any 
compound or technique on the concentration of the allergen in the sample, and even lead to false results. Spurious effects can be recorded using this method, such as an increase in concentration of the allergen in a sample that has been treated with a technique known to reduce the allergen (see for example, [13]). Consequently, a large number of replicates are typically necessary in order to discern any statistically significant difference between samples and controls. This leads to an expense in both time and resources.

Powdered samples are also sensitive to accidental loss to the surrounding environment during handling. Added difficulties arise if the effects of electrostatic techniques, in particular corona discharge, on the allergen are being investigated as described below. When testing novel techniques of destroying harmful allergens in vitro a more reliable method of preparing samples of known concentration is needed.

This paper investigates the best method of preparing allergen samples for the analysis of any effects that corona discharge may have upon the allergen. The variability of Der p1 concentration in samples of sieved, concentrated Dermatophagoides pteronyssinus HDM culture using different methods of application was measured. The effect of negative corona discharge on Der p1 using the heterogeneous Der p1-carrying faecal particles and the new evaporated solution method are also described and compared.

\section{Materials and methods}

\subsection{Preparation of HDM cultures}

Cultures containing Dermatophagoides pterynissinus HDMs were established by using material obtained from British domestic vacuum cleaner bags that tested positive for Der p1. Cultures were maintained on a substrate of finely crushed dog biscuit and dried yeast mixed in a ratio of approximately 30 to 1 [14]. The established HDM culture was then sieved to below $63 \mu \mathrm{m}$.

\subsection{Distributing culture medium particles with a spatula or sieve}

A circular piece of aluminium foil ( $70 \mathrm{~mm}$ diameter) was divided equally into eight sectors. One gram of sieved culture medium was then spread as evenly as possible over the foil using a spatula. The culture medium in the individual sectors was then brushed into an eppendorf tube and 1\% BSA-PBS-T (Bovine Serum Albumin in $0.05 \%$ Phosphate Buffered Saline with Tween 20) was added to produce a 1 in 100 dilution for subsequent testing for Der p1 concentration by a two-site monoclonal antibody enzyme-linked immunosorbent assay [15] (ELISA, from INDOOR Biotechnologies Ltd., Cardiff, UK). This procedure was repeated twice. Results are shown in Fig. 2A.

To test the importance of the dust distribution technique, $1 \mathrm{~g}$ of sieved culture medium was evenly distributed over the foil circles using a fine sieve $(63 \mu \mathrm{m}$ pore 
size), and then removed as above and prepared for ELISA. This was also repeated twice. Results are shown in Fig. 2B.

\subsection{Using an aqueous solution of culture medium}

Another application method employed an aqueous solution of the culture medium. Fifteen grams of the $<63 \mu \mathrm{m}$ culture fraction was added to $400 \mathrm{ml}$ of distilled water. This mixture was stirred well to enable all the Der $\mathrm{p} 1$ to dissolve, then passed through filter paper (Whatman, qualitative, grade 4) to remove solid material. Thimerosal (sodium ethylmercurithiosalicylate) was added at a concentration of $0.001 \%$ to prevent bacterial, fungal and mould growth. Three hundred millilitres of this solution were then poured onto an aluminium foil-covered $736 \mathrm{~cm}^{2}$ tray and dried at $37^{\circ} \mathrm{C}$. Two circular pieces of foil of $70 \mathrm{~mm}$ diameter were then cut out of the foil at random positions, each were divided into eight sectors and placed in an eppendorf. One per cent BSA-PBS-T was added to give a 1 in 200 dilution in preparation for testing by ELISA. Results are shown in Fig. 2C.

The final method of Der p1 application investigated also used the culture medium solution, prepared as above. Sixteen $100 \mu \mathrm{l}$ aliquots of this solution were micropipetted onto foil and left to evaporate in a drying oven at $37^{\circ} \mathrm{C}$. These were diluted 6-fold with 1\% BSA-PBS-T and prepared for ELISA. Results shown in Fig. 2D.

In order to determine whether the act of evaporating the aqueous solution of Der p1 had any detrimental effect on the concentration itself, $12100 \mu 1$ samples were evaporated at $37^{\circ} \mathrm{C}$ and diluted in $1 \%$ BSA-PBS-T as above and then assayed by ELISA. These samples were then compared to the Der pl concentration of $12100 \mu 1$ drops that were placed straight into an eppendorf and diluted 6-fold in 1\% BSAPBS-T without being evaporated.

\subsection{Exposing Der pl-carrying faecal particles to negative continuous glow corona discharge}

A simple point-to-plane corona apparatus was used with a pin with point radius $45 \mu \mathrm{m}$ fixed with an interelectrode gap of $15 \mathrm{~mm}$ above a square, planar aluminium electrode. This is illustrated schematically in Fig. 1. Onto this, $400 \mu \mathrm{g}$ of finely sieved $(<63 \mu \mathrm{m})$ culture medium was placed using a spatula and half of this was then removed, weighed and kept as the control in ambient air for the duration of the experiment. The remaining sample was then distributed evenly over the planar electrode. The pin was connected to a negative d.c. high voltage supply to produce an ionic bombardment equivalent to a corona current of $90 \mu \mathrm{A}$. Samples were exposed for $60,120,180,240$ and $300 \mathrm{~min}$. The sample was then carefully removed, weighed and diluted 100-fold with 1\% BSA-PBS-T in preparation for ELISA. This protocol was followed to produce 12 replicates. Any solid particulates that accumulated on the pin during corona discharge was brushed into an eppendorf and added to $200 \mu 1 \%$ BSA-PBS-T and prepared for ELISA. Results shown in Fig. 3. 


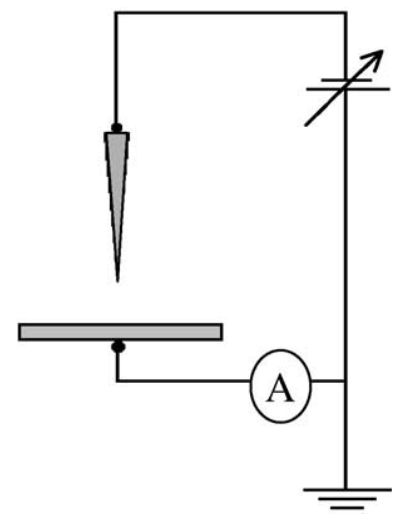

Fig. 1. Schematic diagram of the point-to-plane corona apparatus.

\subsection{Exposing evaporated Der pl solution to negative continuous glow corona discharge}

A solution containing HDM allergen was prepared as described in Section 2.3. Hundred-microlitre samples of this Der $\mathrm{p} 1$ solution were then pipetted onto $20 \mathrm{~mm}^{2}$ squares of aluminium foil. The sample and its paired control were prepared adjacently. These were then dried at $37^{\circ} \mathrm{C}$. The Der p1 sample was then fixed to the planar electrode and exposed to corona discharge as described in Section 2.4. After this exposure time the sample was removed and prepared for ELISA. The controls were exposed to ambient air for the equivalent period of time, diluted 6 -fold in $1 \%$ BSA-PBS-T and then prepared in the same way. This was repeated 6 times for each exposure. Results are shown in Fig. 3.

\subsection{Data analysis}

The concentration data from the solid mixture of culture medium distributed by spatula or sieve (Section 2.2) were expressed as $\mu \mathrm{g} \mathrm{g}^{-1}$ of culture medium, and the data from the tests using culture medium solution (Section 2.3) were expressed as $\mathrm{ng} \mathrm{m}{ }^{-1}$. The variability of the samples was then compared using the coefficient of variation $(\mathrm{CV})$, which is the ratio of the standard deviation to the mean, expressed as a percentage.

For each duration of exposure to corona (Sections 2.4 and 2.5), the Der p1 concentrations of the exposed samples were compared to their paired controls by the $F$-test. The concentration data for each time period was then analysed using the Paired $t$-test for means, or the Mann Whitney-U test where appropriate. Statistical significance was defined as $P<0.05$. These concentration data were then presented as mean percentage reductions in Der $\mathrm{p} 1$ concentration calculated by comparing the Der p1 sample to its equivalent control in the specific time period. 


\section{Results}

\subsection{Concentration homogeneity}

The Der $\mathrm{p} 1$ concentration can be seen to vary considerably in all tests using sieved culture medium. Fig. 2 shows the Der p1 concentration of the individual samples taken from the sectors of the foil and also of the aliquots of culture medium solution. Fig. 2A shows the concentration of sectors with allergen sample distributed with a spatula. The concentration of most sectors varied from 32.45 to $99.63 \mu \mathrm{gg}^{-1}$ but sectors 7 and 8 had a much greater concentration of 604.81 and $618.81 \mu \mathrm{g} \mathrm{g}^{-1}$, respectively.
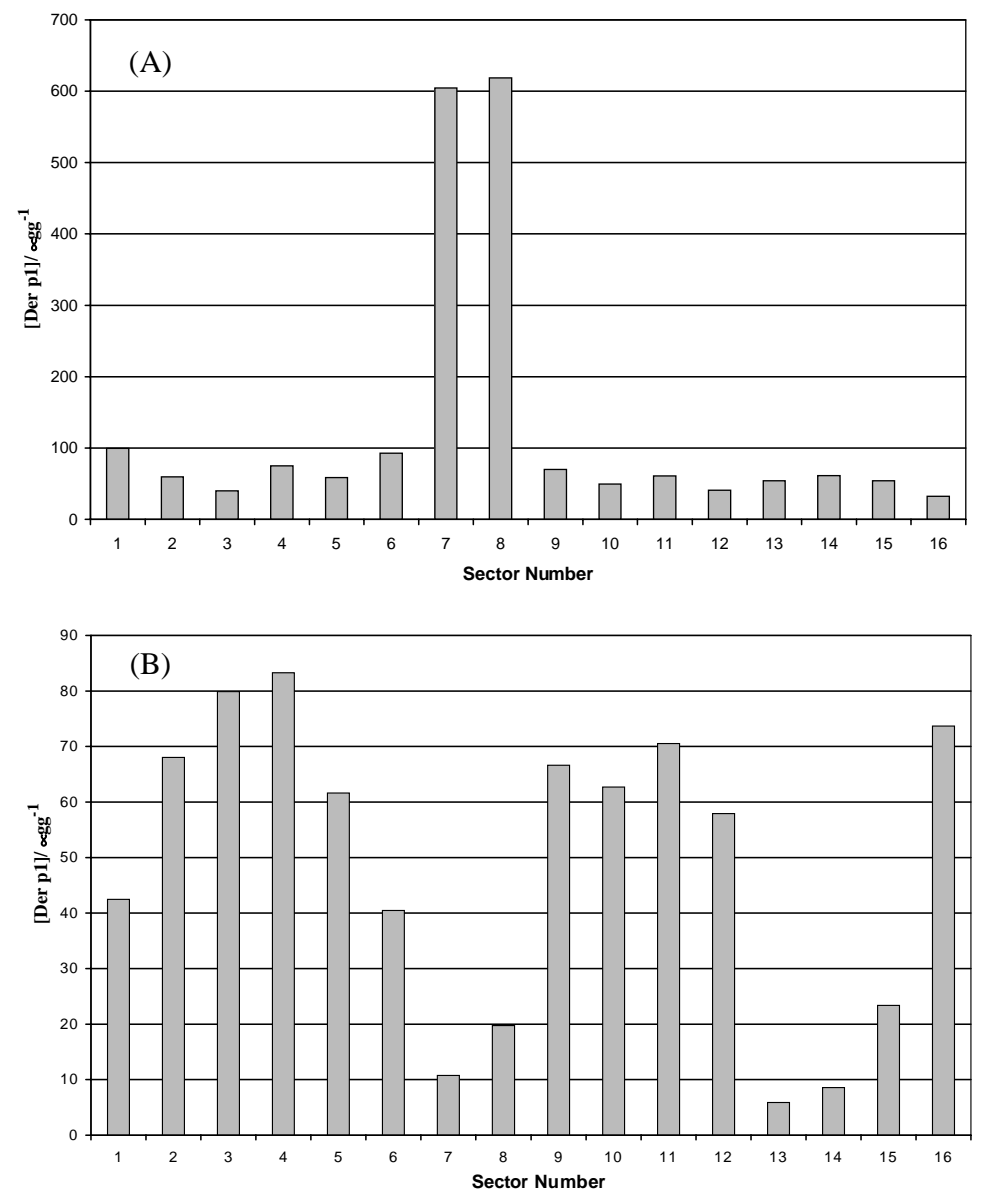

Fig. 2. The concentration of Der p1 in the individual samples when distributed using various methods of application. (A) Using a spatula to distribute the sieved culture; (B) using a $<63 \mu \mathrm{m}$ pore sieve; (C) using a Der pl solution evaporated onto a tray; (D) using $100 \mu \mathrm{l}$ aliquots, notice the uniformity of concentration in these samples compared to the other methods above. 

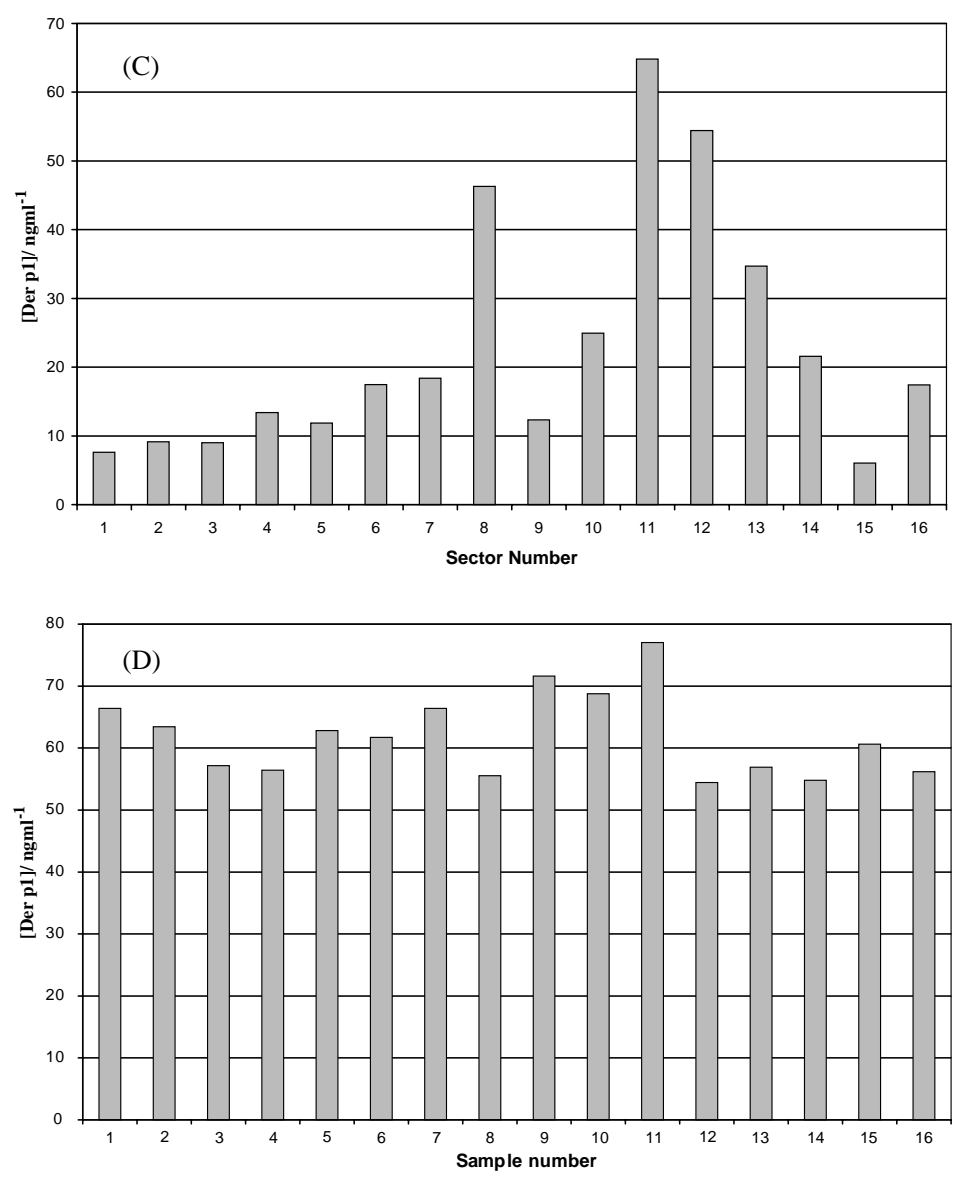

Fig. 2. (Continued).

Using a sieve to spread the allergen samples (see Fig. 2B) led to Der p1

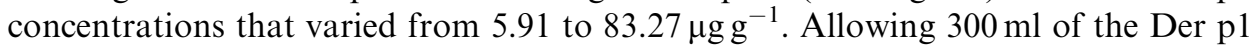
solution to evaporate on foil led to a greater variability in concentration than using a sieve to distribute solid particles (see Fig. 2C). The Der p1 concentration varied from 6.04 to $64.80 \mu \mathrm{gg}^{-1}$.

However, using the evaporated $100 \mu \mathrm{l}$ drops of Der p1 solution led to a very uniform concentration throughout the samples tested (see Fig. 2D). The concentration of samples varied from 54.43 to $77.03 \mathrm{ng} \mathrm{ml}^{-1}$; a range of only $22.60 \mathrm{ng} \mathrm{ml}^{-1}$ which is an acceptable variability and corresponds to the uniform nature of homogeneous solutions. Table 1 gives the coefficient of variation between sectors for each of the methods of sample application. These values show that the concentration variability of a Der p1 sample distributed by a spatula is the greatest followed by the evaporating $300 \mathrm{mls}$ of Der pl solution onto a tray method, using a sieve and the 
Table 1

The coefficient of variation of the samples' concentration taken from the experiments using different methods of Der p1 distribution

\begin{tabular}{lllll}
\hline Method of sample application & Spatula & Sieve & Tray & Aliquot \\
\hline CV & 146.07 & 55.55 & 77.04 & 10.82 \\
\hline
\end{tabular}

aliquot method varied least. The $100 \mu \mathrm{l}$ aliquot method gave a concentration variation of more than 10 -fold less than using a spatula to distribute the sample. The results from the experiment to determine whether evaporation reduced the concentration of Der p1 showed no difference between the two sets of samples.

\subsection{Effect of corona discharge on Der p1}

Fig. 3 shows that exposure of evaporated Der p1 samples to negative corona discharge (see Section 2.5) led to statistically significant amounts of Der p1 being denatured with six replicates of each time point $(P<0.01$, using the Paired $t$-test for means). The percentage reductions in Der p1 concentration increased from $37.26 \pm 6.47 \%$ after $60 \mathrm{~min}$ of exposure through $39.80 \pm 7.49 \%, 43.22 \pm 7.07 \%$, $55.81 \pm 7.58 \%$ and $67.37 \pm 5.78 \%$ reduction after $120,180,240$ and 300 min exposure to negative continuous glow corona, respectively.

Similar to the results obtained from using evaporated Der p1 samples, the mean percentage reductions in the Der p1 concentration of dry culture medium (see Section 2.4) increased with each increase in exposure time (see Fig. 3). The percentage reductions increased from $0.8 \pm 11.05 \%$ after $60 \mathrm{~min}$ exposure through $4.11 \pm 7.07 \%, 19.83 \pm 9.45 \%, 21.22 \pm 12.46 \%$ and $26.86 \pm 14.64 \%$ after $120,180,240$ and $300 \mathrm{~min}$ exposure, respectively.

However, as would be expected from the large standard errors of these results, these reductions were not statistically significant. This reinforces the argument that the use of heterogeneous powder mixtures inevitably leads to concentration variability and difficulties in distinguishing the effects of any technique upon the sample.

Another problem encountered during corona discharge on the dry culture medium was the loss of sample to the surrounding environment. Electrostatic charging and field effects led to a visible accumulation of solid material on the upper electrode. The total amount was small, weighing $<1 \mathrm{mg}$. However, by adding this to $200 \mu \mathrm{l}$ of $1 \%$ BSA-PBS-T, the ELISA determined that the sample consisted of $23.92 \mathrm{ng}$ of Der p1.

\section{Discussion}

This investigation has demonstrated that the inherent heterogeneity of sieved, allergen containing samples causes significant variability in data obtained from 


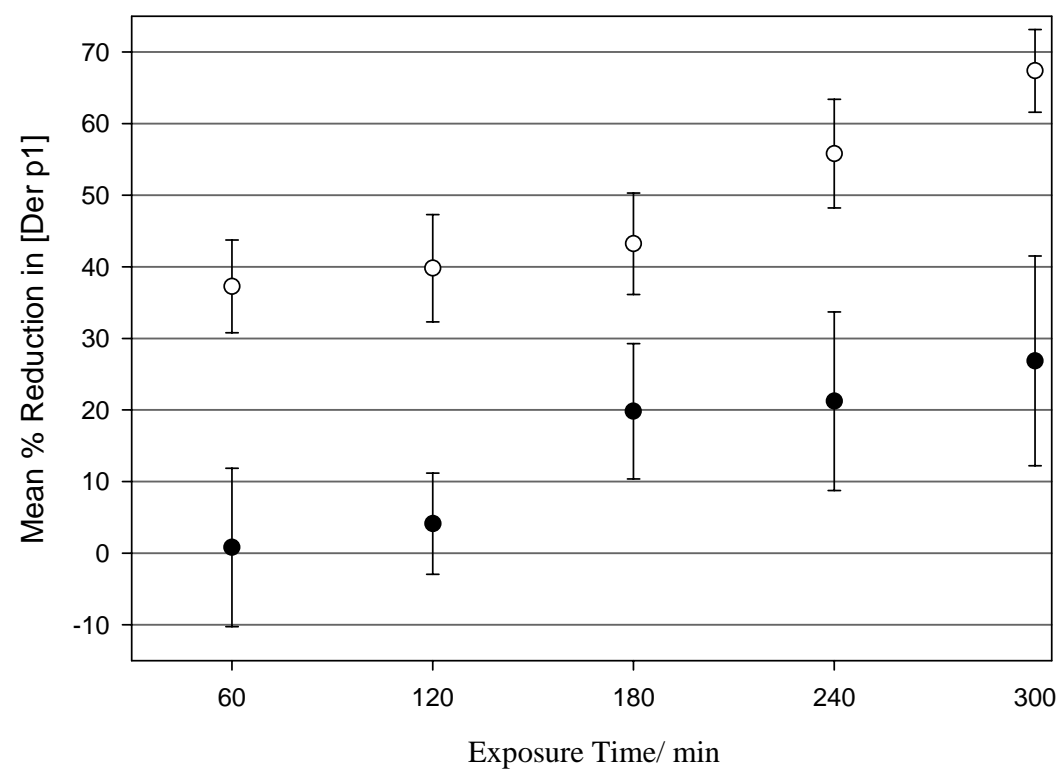

Fig. 3. The mean percentage reduction in Der $\mathrm{p} 1$ concentration after irradiation by negative continuous glow corona on sieved culture medium $(\bullet)$, or aliquots of evaporated Der pl solution $(\bigcirc)$. Culture medium: $n=12$; evaporated solution: $n=6$; SEM shown.

them. Additional problems encountered when using a powdered sample in the electrostatic investigations demonstrated here were the loss of material to the atmosphere and electrodes. The particles of the powder mixture on the planar electrode became electrostatically charged as a consequence of exposure to uni-polar ions. Particle movement within the interelectrode gap was subsequently induced by the electric field. This explains the behaviour of the powder escaping the corona setup or being attracted to the negative polarity of the upper electrode. The allergen could also have been attracted to the opposite polarity of the upper electrode because of its inherent net positive polarity in dry conditions [7]. These artefacts caused the 12 replicates to be insufficient in obtaining a statistically significant value for the amount of Der p1 denatured.

The use of single, small aliquots taken from a dissolved sample has been shown to overcome these problems and is ideal for investigating the effect of electrostatic techniques on protein samples. Consequently, the consistency and reliability of allergen denaturing tests can be substantially improved. The sample is firmly attached to the planar electrode and so will not be lost to the atmosphere, nor migrate to the upper electrode. This method also allows different electrode configurations to be tested such as wire-to-cylinder and wire-to-plane as the samples may also be placed vertically or inverted. This method of allergen preparation would also be suitable for future experimental procedures for evaluating the effects of electric fields on allergens. 
With just six replicates for each time of exposure of evaporated samples to negative glow corona, all percentage reductions in Der p1 concentration were significant and with small variation about the mean. The percentage reductions observed in the concentration of Der p1 in the evaporated samples were considerably higher than those observed with the sieved culture. This could be due to a number of factors, such as loss of non-Der p1 material through handling the powder material or through electrostatic attraction to the pins. When using a pin-to-plane corona arrangement, the point electrode became thinly coated with the allergenic sample. This would further alter the allergen concentration of the sample on the planar electrode giving a false reduction in the final analysis. Another possibility is that the non-allergenic particles of the solid mixture physically protect the Der $\mathrm{p} 1$ molecule from exposure to the corona products. Research is ongoing into the possibility of a relationship between the concentration of the sample and the percentage reduction observed.

The tests reported here concentrated on the Der p1 allergen specifically. Other dust mite allergens including Der p2, Der f1 and the major cat allergen Fel d1 are currently under investigation using the same allergen preparation technique [16]. With the discovery that corona discharge can destroy Der p1 and other allergens, intense research efforts will be focussed into producing an optimised technique and protocol for applying corona discharge to destroy allergens in the domestic environment. The improved method for preparing allergen samples detailed here will aid these efforts.

\section{Acknowledgements}

The support of the National Asthma Campaign, who funded this work, is gratefully acknowledged.

\section{References}

[1] P.D. Phelan, Asthma in children: epidemiology, BMJ 308 (1994) 1584-1585.

[2] H. Harving, J. Korsgaard, R. Dahl, Clinical efficacy of reduction in house-dust-mite exposure in specially designed mechanically ventilated healthy homes, Allergy 49 (1994) 866-880.

[3] J.K. Peat, R.H. van den Berg, W.F. Green, C.M. Mellis, S.R. Leeder, A.J. Woolcock, Changing prevalence of asthma in Australian children, BMJ 308 (1994) 1591-1596.

[4] J. Korsgaard, House-dust mites and asthma. A review on house-dust mites as a factor for mite allergy, Allergy 53 (Suppl. 48) (1998) 77-83.

[5] S.J. Thompson, F. Carswell, The major allergen of the house dust mite, Dermatophagoides pteronyssinus is synthesised and secreted into its alimentary canal, Int. Arch. Allergy. App. Immun. 85 (1988) 312-315.

[6] E.R. Tovey, M.D. Chapman, T.A.E. Platts-Mills, Mite faeces are a major source of house dust mite allergens, Nature 289 (1981) 592-593.

[7] P.T. Gaynor, J.F. Hughes, Electrostatic charge characteristics of Der p1 alleren-carrying particles and the house dust mite Dermatophagoides pteronyssinus, Med. Biol. Eng. Comput. 36 (1998) 351-354. 
[8] K.Y. Chua, G.A. Stewart, W.R. Thomas, R.J. Simpson, R.J. Dilworth, T.M. Plozza, K.J. Turner, Sequence analysis of cDNA coding for a major house dust mite allergen, Der p1. Homology with cysteine proteases, J. Exp. Med. 167 (1988) 175-182.

[9] M.J. Colloff, J. Ayres, F. Carswell, P.H. Howarth, T.G. Merrett, E.B. Mitchell, M.J. Walshaw, J.O. Warner, J.A. Warner, A.A. Woodcock, The control of allergens of dust mites and domestic pets: a position paper, Clin. Exp. Allergy 22 (Suppl 2) (1992) 1-28.

[10] I. Ben Dov, I. Amiraw, M. Sochina, I. Amitari, E. Bar Yishay, S. Godfrey, Effect of negative ionisation of inspired air on the response of asthmatic children to exercise and inhaled histamine, Thorax 38 (1983) 584-588.

[11] D.B. Peden, R.W. Setzer Jr., R.B. Devlin, Ozone exposure has both a priming effect on allergeninduced responses and an intrinsic inflammatory action in the nasal airways of perennially allergic asthmatics, Am. J. Respir. Crit. Care Med. 151 (1995) 1336-1345.

[12] N. Goodman, J.F. Hughes, The effect of corona discharge on Der p1. Clin. Exp. Allergy, in press.

[13] M.J. Colloff, C. Taylor, T.G. Merrett, The use of domestic steam cleaning for the control of house dust mites, Clin. Exp. Allergy 25 (1995) 1061-1066.

[14] G.W. Wharton, House dust mites, J. Med. Ent. 12 (1976) 577-621.

[15] C.M. Luczynska, L.K. Arruda, T.A.E. Platts-Mills, J.D. Miller, M. Lopez, M.D. Chapman, A twosite monoclonal antibody ELISA for the quantification of the major Dermatophagoides spp. Allergens, Der p I and Der f I, J. Immunol. Meths. 118 (1989) 227-235.

[16] N. Goodman, J.F. Hughes, unpublished data. 